\title{
Catechol enhances chemo- and radio-sensitivity by targeting AMPK/Hippo signaling in pancreatic cancer cells
}

\author{
JEONG YONG MOON ${ }^{1}$, MERAN KESHAWA EDIRIWEERA ${ }^{1}$, JI YEON RYU ${ }^{2}$, \\ HEE YOUNG $\mathrm{KIM}^{3}$ and SOMI KIM $\mathrm{CHO}^{1-4}$ \\ ${ }^{1}$ Subtropical/Tropical Organism Gene Bank; ${ }^{2}$ School of Biomaterials Science and Technology, \\ College of Applied Life Sciences; ${ }^{3}$ Interdisciplinary Graduate Program in Advanced Convergence Technology and Science; \\ ${ }^{4}$ Faculty of Biotechnology, College of Applied Life Sciences, SARI, Jeju National University, \\ Jeju Special Self-Governing Province 63243, Republic of Korea
}

Received April 17, 2020; Accepted November 26, 2020

DOI: 10.3892/or.2021.7924

\begin{abstract}
Overcoming chemo- and radio-resistance is a major challenge in pancreatic cancer treatment. Therefore, there is an urgent need to discover novel therapeutic approaches to avoid chemo- and radio-resistance in pancreatic cancer. Catechol is a phytochemical found in some fruits and vegetables. A few studies have reported on the potential anticancer effects of pure catechol. The present study aimed to explore the chemo- and radio-sensitizing effects of catechol in Panc-1 human pancreatic cancer cells. The effects of catechol on Panc-1 cell proliferation, clonogenic survival, invasion, and migration were assessed using MTT, cell migration, and Transwell invasion assays. The chemo- and radio-sensitizing effects of catechol on Panc-1 cells were evaluated via MTT assay and flow cytometry. Western blotting was conducted to analyze the expression of proteins involved in several mechanisms induced by catechol in Panc-1 cells, including growth inhibition, apoptosis, suppression of epithelial-mesenchymal transition (EMT), and chemo- and radio-sensitizing activities. The results indicated that catechol inhibited proliferation, promoted apoptosis, and suppressed cell migration, invasion, and EMT in Panc-1 cells in a dose-dependent manner. Catechol
\end{abstract}

Correspondence to: Professor Somi Kim Cho, School of Biomaterials Science and Technology, College of Applied Life Sciences, Jeju National University, 102 Jejudaehak-ro, Jeju-si, Jeju Special Self-Governing Province 63243, Republic of Korea E-mail: somikim@jejunu.ac.kr; phd.kim.somi@gmail.com

Abbreviations:AMPK,AMP-activated protein kinase; $\mathrm{CI}$,combination index; CTGF, connective tissue growth factor; CYR61, cysteine-rich angiogenic inducer 61; DMEM, Dulbecco's modified Eagle's medium; EMT, epithelial-mesenchymal transition; FBS, fetal bovine serum; MTT, 3-(4,5-dimethylthiazol-2-yl)-2,5-diphenyltetrazoliumbromide; PARP, poly(ADP-ribose) polymerase; PI, propidium iodide; YAP, yes-associated protein

Key words: AMPK/Hippo signaling, catechol, chemosensitivity, pancreatic cancer, radiosensitivity treatment also induced the phosphorylation of AMP-activated protein kinase (AMPK) with a concomitant reduction in the expression of Hippo signaling pathway components, including Yes-associated protein, cysteine-rich angiogenic inducer 61, and connective tissue growth factor. In addition, catechol enhanced the chemosensitivity of Panc-1 cells to gemcitabine, a commonly used chemotherapy in pancreatic cancer treatment. A combination of catechol and radiation enhanced apoptosis and increased the expression of two radiation-induced DNA damage markers, p-ATM and p-Chk2. Collectively, the present results demonstrated that catechol, a naturally occurring compound, could suppress the proliferation of pancreatic cancer cells, reduce the expression of EMT-related proteins, and enhance the chemo- and radio-sensitivity of Panc-1 cells by targeting AMPK/Hippo signaling.

\section{Introduction}

Worldwide, pancreatic cancer is the seventh major cause of cancer-related deaths in both males and females (1). Economically developed countries experience a higher incidence of pancreatic cancer cases and higher mortality rates than developing countries (2). In the United States, it was estimated that by the end of 2019 , there would be 56,770 new pancreatic cancer cases (males: 29,940; females: 26,830), and 45,750 pancreatic cancer-related deaths (males: 23,800; females: 21,950) (2). Despite the developments in screening methods and advances in cancer drug discovery approaches, new pancreatic cancer cases are predicted to rise within the next decade (3). Based on histological and molecular characteristics, pancreatic cancer can be classified into two major subtypes, namely, exocrine (pancreatic adenocarcinoma) and endocrine tumors (3). Although molecular and histological subtyping helps to determine treatment plans in other human cancers, treatment plans for pancreatic cancer do not necessarily depend on the pancreatic cancer subtype (4). Radiotherapy, chemotherapy, and surgery are currently used to extend the survival of pancreatic cancer patients $(3,5)$. However, radiotherapy and chemotherapy treatments are ineffective for advanced-stage pancreatic cancer. Gemcitabine is a commonly used chemotherapy for all stages of pancreatic cancer $(6,7)$. Development of resistance to gemcitabine has been identified as 
a major clinical barrier in pancreatic cancer treatment, thereby limiting the clinical efficacy of this drug. Surgical removal of the pancreas or surrounding metastatic tumors has been reported to cause some digestive issues and functional loss in pancreatic cancer patients (8). Therefore, there is an urgent necessity to explore new anticancer therapeutics, which can effectively target pancreatic cancer cells without causing adverse side effects.

Various plant extracts and plant-derived secondary metabolites have been used to treat a number of diseases and have been reported to exert a range of pharmacological effects (9). In recent anticancer drug discovery approaches, great effort has been invested in attempts to identify plant-derived secondary metabolites as novel anticancer therapeutics as they exert favorable therapeutic efficacies over synthetic anticancer drugs (10). Catechol (pyrocatechol) is a secondary metabolite found in some fruits and vegetables $(11,12)$. Catechol is chemically synthesized in large quantities to be used as a precursor in the pharmaceutical industry. Some phytochemicals with a catechol moiety, such as fisetin, luteolin, procyanidin B2, quercetin, and 7,3',4'-trihydroxyisoflavone have been revealed to exert anticancer effects in several in vitro models (12). The anticancer efficacy of pure catechol has been reported in lung cancer and breast cancer stem cells $(11,12)$. However, to the best of our knowledge, in vitro anticancer effects of pure catechol in pancreatic cancer cells have not been explored yet. Therefore, the present study aimed to explore the possible anti-carcinogenic and radio-sensitizing effects of catechol in pancreatic cancer cells in-vitro.

\section{Materials and methods}

Materials. Cell culture reagents used in this investigation were purchased from Invitrogen; Thermo Fisher Scientific, Inc. All other chemicals used were purchased from Sigma-Aldrich; Merck KGaA unless otherwise stated. Primary antibodies [Bax (product no. 2774), Bcl-2 (product no. 2872), cleaved caspase-3 (product no. 9664), cleaved poly(ADP-ribose) polymerase (PARP) (cat. no. 5625S), GAPDH (product no. 5174S), phosphorylated-signal transducer and activator of transcription 3 (p-STAT3) (product no. 9145S), STAT-3 (product no. 9139S), matrix metalloproteinase-2 (MMP2) (product no. 13132S), Snail (product no. 3879S), vimentin (product no. 5741S), p-AMP-activated protein kinase (p-AMPK $\alpha)$ (product no. 2535S), AMPK $\alpha$ (product no. 2532S), Yes-associated protein (YAP) (product no. 4912S), cysteine-rich angiogenic inducer 61 (Cyr61) (product no. 14479S), connective tissue growth factor (CTGF) (product no. 10095S), p-ataxia telangiectasia mutated kinase (p-ATM) (product no. 13050S), and p-checkpoint kinase 2 (p-Chk2) (product no. 2661S) were purchased from Cell Signaling Technology. The primary antibody MMP9 was purchased from EMD Millipore (product no. AB19016). Secondary antibodies [goat anti-rabbit (cat.no. PI-1000-1) and horse anti-mouse immunoglobin (Ig)G (cat. no. PI-2000-1)] were purchased from Vector Laboratories, Inc. The BS ECL Plus kit (cat. no. W6002) was purchased from Biosesang, Inc.

Cell culture. Four human pancreatic cancer cell lines, Aspc-1, MiaPaCa2, Panc-1, and SNU-213, were obtained from the laboratory of Professor Jae-Hoon Kim at the Faculty of
Biotechnology, Jeju National University, Republic of Korea. Cells were maintained in Dulbecco's modified Eagle's medium (DMEM) supplemented with $10 \%$ fetal bovine serum and an antibiotic cocktail $(100 \mu \mathrm{g} / \mathrm{ml}$ streptomycin and $100 \mathrm{U} / \mathrm{ml}$ penicillin) and incubated at $37^{\circ} \mathrm{C}$ under $5 \% \mathrm{CO}_{2}$. Dermal fibroblasts (13) were cultured in DMEM under similar culture conditions. Cells were sub-cultured upon $80 \%$ confluency.

Cell viability assay. The 3-(4,5-dimethylthiazol-2-yl)-2,5-diphenyltetrazoliumbromide (MTT) assay was used as the cell viability assay in the present investigation. Prior to the MTT assay, four types of pancreatic cancer cells (Aspc-1, MiaPaCa2, Panc-1 and SNU-213) were cultured in 96-well culture plates $\left(5 \times 10^{3}\right.$ cells/well) and incubated for $48 \mathrm{~h}$ at $37^{\circ} \mathrm{C}$. Following incubation, the cells were exposed to different doses $(12.5-200 \mu \mathrm{M})$ of catechol and incubated again for $48 \mathrm{~h}$ at $37^{\circ} \mathrm{C}$. In chemosensitivity evaluation experiments of catechol, Panc-1 cells were co-treated with catechol $(50$ or $100 \mu \mathrm{M})$ and gemcitabine $(0.25-1 \mu \mathrm{M})$ for $48 \mathrm{~h}$. The MTT assay was then conducted to assess cell viability. Dimethyl sulfoxide (DMSO) was used to dissolve purple formazan and the absorbance was recorded at $540 \mathrm{~nm}$ using a micro-plate reader (Sunrise; Tecan Group, Ltd.). The percentage of cell viability was determined using the formula: $\left[\left(A_{T}-A_{B}\right) /\left(A_{C}-A_{B}\right)\right] \times 100$, where $A_{T}$ denotes the absorbance value of the treated group, $A_{B}$ denotes the absorbance value of the blank, and $\mathrm{A}_{C}$ denotes the absorbance value of the untreated control. GraphPad Prism 7.00 (GraphPad Software, Inc.) was used to determine the half maximum inhibitory concentration $\left(\mathrm{IC}_{50}\right)$ of catechol in each pancreatic cancer cell line.

Colony formation assay. Prior to the colony formation assay, Panc-1 cells (200 cells/dish) were seeded in cell culture dishes and exposed to catechol alone, or to a combination of catechol $(50 \mu \mathrm{M})$ and ionizing radiation (2 or $4 \mathrm{~Gy}$ ). After 10 days, the cells were fixed with $4 \%$ formaldehyde at room temperature for $30 \mathrm{~min}$, stained with crystal violet ( $0.5 \%$ crystal violet), incubated at room temperature for $30 \mathrm{~min}$ and washed. NIST's Integrated Colony Enumerator software (https://www.nist.gov/services-resources/software/nists-integrated-colony-enumerator-nice) was used to count the number of colonies in the culture dishes. Irradiation was carried out at the Applied Radiological Science Institute at Jeju National University using a ${ }^{60} \mathrm{CO}$ Theratron-780 teletherapy unit.

Wound healing assay. Panc-1 cells (1x10 $/$ well) were cultured in 6-well plates and incubated until the cells reached confluence. A uniform scratch was then made through the cell monolayer using a sterilized $200-\mu 1$ pipette tip. After washing with phosphate-buffered saline (PBS), different doses (25 or $50 \mu \mathrm{M})$ of catechol were added to the wells with DMEM containing 5\% FBS (14), and the cells were incubated for $24 \mathrm{~h}$. Following incubation, the width of the wound was measured under a phase-contrast microscope (x100).

Invasion assay. Prior to the invasion assay, cells were exposed to different concentrations $(12.5,25$, or $50 \mu \mathrm{M})$ of catechol and incubated for $24 \mathrm{~h}$. For this assay, a Transwell system (24-well plate; $0.2 \mu \mathrm{m}$ pore; Corning, Inc.) was used. The 
upper chambers were coated with $1 \%$ Matrigel by incubating at $37^{\circ} \mathrm{C}$ for $1 \mathrm{~h}$. The upper chambers received $200 \mu \mathrm{l}$ of DMEM and $1 \times 10^{5}$ cells (without FBS) supplemented with or without catechol $(12.5,25$ and $50 \mu \mathrm{M})$, while the lower chambers received DMEM supplemented with 10\% FBS. Following $24 \mathrm{~h}$ of incubation at $37^{\circ} \mathrm{C}$, invading Panc- 1 cells were first fixed with methanol and then $4 \%$ paraformaldehyde at room temperature for $30 \mathrm{~min}$, stained with $2 \%$ crystal violet for $30 \mathrm{~min}$ at room temperature, washed with distilled water and observed under a phase contrast microscope (x100).

Hoechst staining. Panc-1 cells $\left(5 \times 10^{4} /\right.$ well) were cultured on coverslips and incubated for $24 \mathrm{~h}$. Following incubation cells were exposed to catechol for $48 \mathrm{~h}$. Cells were then fixed with $4 \%$ formaldehyde for $30 \mathrm{~min}$ at room temperature and stained with Hoechst 33342 solution $(0.01 \mathrm{mg} / \mathrm{ml})$ for $30 \mathrm{~min}$ at room temperature. Stained Panc-1 cells were observed and images were captured under a fluorescence microscope (x100) (IX73; Olympus Corporation).

Flow cytometry. The effects of catechol on the cell cycle and apoptosis were investigated using flow cytometry at the Bio-Health Materials Core-Facility at Jeju National University. Flow cytometric analysis was performed with BD FACSDiva ${ }^{\mathrm{TM}}$ Software (BD Biosciences). Prior to the analysis, the cells $\left(3 \times 10^{4}\right.$ cells) were washed twice with PBS and fixed in $70 \%$ ethanol for $30 \mathrm{~min}$ at $37^{\circ} \mathrm{C}$. Following fixation, the cells were treated with RNase A $(25 \mathrm{ng} / \mathrm{ml})$ and staining with propidium iodide $(40 \mu \mathrm{g} / \mathrm{ml})$ and subjected to cell cycle analysis. The Annexin V-FITC Apoptosis Detection Kit (BD Biosciences) was used according to the manufacturer's instructions for apoptosis analysis.

Western blot analysis. Following treatments, radioimmunoprecipitation assay buffer (RIPA; Thermo Fisher Scientific, Inc.) was used to extract cell lysates. The protein concentrations were determined using a BCA assay. Upon quantification of proteins, $40 \mu \mathrm{g}$ of proteins were electrophoresed (10-12.5\% gels) and transferred to PVDF membranes. After blocking with $5 \%$ dried non-fat milk overnight at $4{ }^{\circ} \mathrm{C}$, membranes were exposed to different primary antibodies overnight at $4^{\circ} \mathrm{C}$. Most of the primary antibodies and the horseradish peroxidase-conjugated (HRP) goat anti-rabbit IgG secondary antibodies were diluted prior to the experiments (primary antibodies to $1: 1,000$ and secondary antibodies to $1: 5,000$ ). GAPDH or $\beta$-actin (reference protein) was diluted to $1: 10,000$ prior to the experiments. Protein bands were detected using the BS ECL-Plus kit (Biosesang, Inc.), and the ImageJ software (version 1.48; National Institutes of Health) was used to quantify bands.

Statistical analysis. Group comparisons were performed using the paired Student's t-test and one-way analysis of variance (with Duncan's post hoc test) with SPSS software (ver. 18.0; IBM Corp.). P-values <0.05 were considered to indicate a statistically significant difference.

\section{Results}

Catechol inhibits proliferation and induces apoptosis in Panc-1 cells. The MTT assay was used to evaluate the effects of catechol on pancreatic cancer cell proliferation. As revealed in Fig. 1A, catechol inhibited the proliferation of pancreatic cancer cell lines (Aspc-1, MiaPaCa-2, Panc-1 and SNU-213) in a dose-dependent manner at $48 \mathrm{~h}$ post-incubation. Catechol had the greatest anti-proliferative effect $\left(\mathrm{IC}_{50}: 91.71 \pm 5.14 \mu \mathrm{M}\right)$ on Panc-1 cells among the four pancreatic cancer cell lines tested. As catechol exerted the strongest anti-proliferative effect on Panc-1 cells, this cell line was selected for other experiments in the present study. Moreover, it has been demonstrated that Panc-1 cells are resistant to gemcitabine, a commonly used chemotherapeutic drug in pancreatic cancer treatments, compared to Aspc-1 cells (15). Catechol displayed an $\mathrm{IC}_{50}$ value of $162.6 \pm 15.6 \mu \mathrm{M}$ in dermal fibroblasts following $48 \mathrm{~h}$ of exposure (Fig. S1). It was then assessed whether the anti-proliferative effects of catechol were associated with Panc-1 cell cycle arrest. As revealed in Fig. 1B, catechol treatment caused the sub-G1 population to increase, from $4.27 \pm 0.31 \%(0 \mu \mathrm{M})$ to $16.33 \pm 1.13 \%(200 \mu \mathrm{M})$. Moreover, as revealed in Fig. 1C, morphological changes associated with apoptosis such as condensed and fragmented chromatin and apoptotic bodies (arrows) were evident in catechol-treated (50, 100 and $200 \mu \mathrm{M})$ Panc-1 cells. Next, we assessed the effects of catechol on the expression of apoptosis-associated proteins (cleaved PARP, caspase-3, and cleaved caspase-3) and STAT3 via western blot experiments. The data revealed that catechol increased cleaved PARP and cleaved caspase-3 protein levels, confirming the apoptosis-inducing effects of catechol on Panc-1 cells (Fig. 1D). Catechol also decreased the phosphorylation of STAT3 in a dose-dependent manner (Fig. 1D). The transcription factor STAT3 has been reported to play a key role in tumorigenesis (11). This observation is also consistent with findings from a previous study, which reported that catechol inhibited STAT3 activation in breast cancer stem cells (11). Collectively, the results demonstrated that catechol inhibited the proliferation of Panc-1 cells and promoted apoptosis through the inactivation of STAT3, one of the known targets of apoptosis (16).

Catechol suppresses EMT. Next, two marginally cytotoxic doses $(12.5$ and $50 \mu \mathrm{M})$ of catechol were selected for migration and invasion assays to examine whether catechol could suppress EMT in Panc-1 cells. The results of the migration and invasion assays revealed that catechol reduced cell migration (Fig. 2A) and invasion (Fig. 2B) in Panc-1 cells at $24 \mathrm{~h}$ post-incubation. Furthermore, catechol treatment caused an increase in the expression levels of E-cadherin protein along with a gradual decrease in the expression of two EMT markers, Snail and vimentin, in Panc-1 cells (Fig. 2C). MMP2 expression was also decreased following catechol exposure in Panc-1 cells (Fig. 2C). The expression of MMP9 was slightly affected by catechol exposure (Fig. 2C). These data indicated that catechol suppressed Panc-1 cell migration and invasion by reducing the expression of EMT-related proteins.

Catechol inhibits the AMP-activated protein kinase (AMPK)/Hippo signaling pathway. The Hippo signaling pathway has been reported to drive tumorigenesis and play a role in developing chemo- and radio-resistance in a range of human cancers (17). YAP is a transcription co-activator in the Hippo signaling pathway (17), and both CYR61 and CTGF 

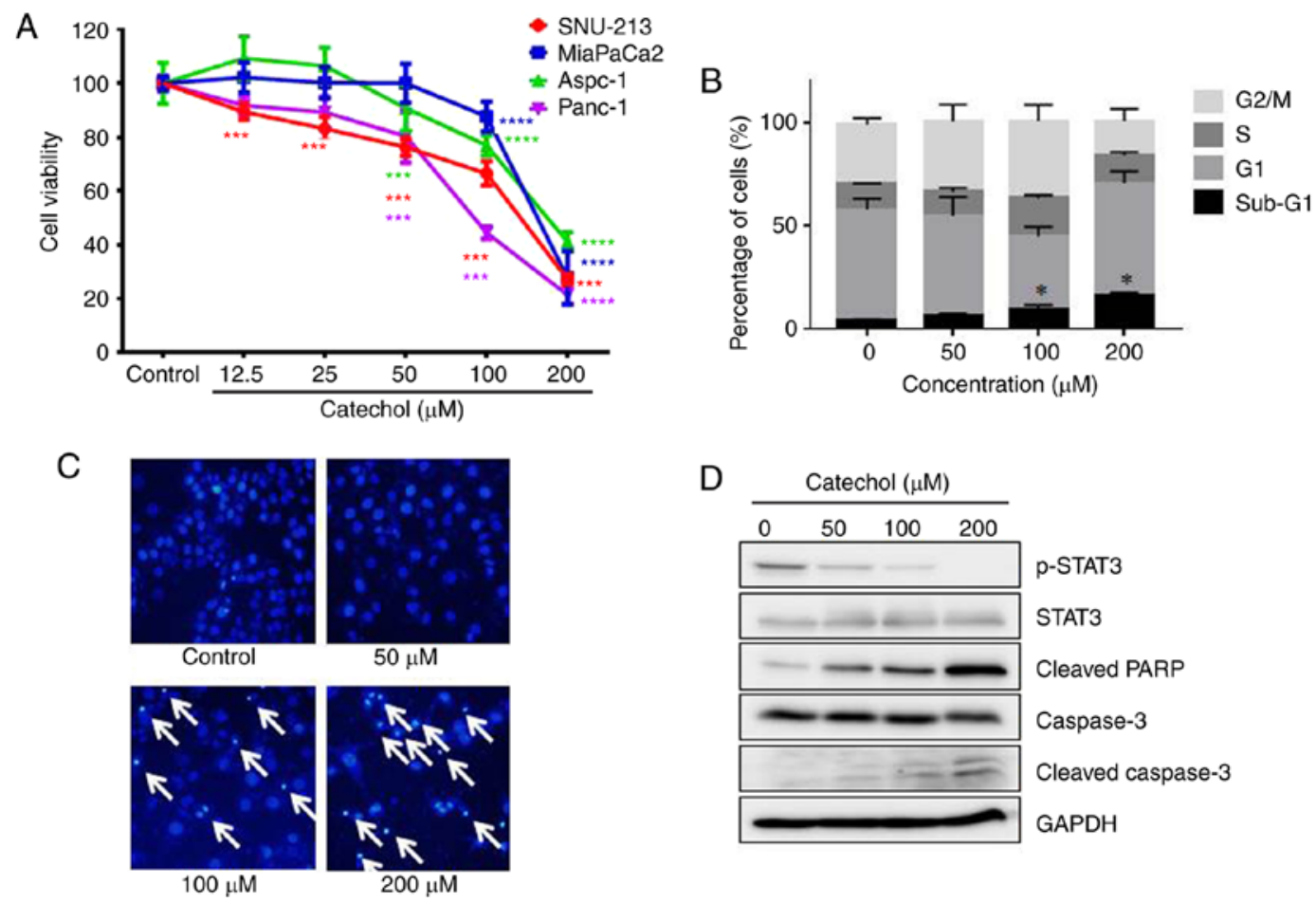

Figure 1. Catechol induces apoptosis in Panc-1 cells. (A) Effects of catechol on the viability of human pancreatic cancer cells. (B) Analysis of the Panc-1 cell cycle after catechol treatment. (C) Panc-1 cells were stained with Hoechst 33342, and nuclear condensation was observed using fluorescence microscopy. (D) Protein levels were examined using western blot analysis with GAPDH as the loading control. Values represent the means $\pm \mathrm{SDs}(\mathrm{n}=3)$. ${ }^{*} \mathrm{P}<0.05,{ }^{* * *} \mathrm{P}<0.001$ and ${ }^{* * * * *} \mathrm{P}<0.0001$ vs. the control. SDs, standard deviations; $\mathrm{p}-$, phosphorylated; PARP, poly(ADP-ribose) polymerase.

are major downstream targets of YAP (18). In addition, activated AMPK has been reported to be associated with YAP inhibition (19). Therefore, the regulatory effects of catechol on the AMPK/Hippo signaling pathway in Panc-1 cells were investigated. It was observed that catechol induced the phosphorylation of AMPK in a dose-dependent manner with a concomitant decrease in YAP, CYR61, and CTGF protein levels (Fig. 3), indicating that catechol could effectively target oncogenic Hippo signaling in Panc-1 cells.

Catechol enhances the chemosensitivity of Panc-1 cells to gemcitabine. To assess whether catechol enhances the in vitro cytotoxic efficacy of gemcitabine. Cell viability was then analyzed using an MTT assay. Combining catechol and gemcitabine resulted in the reduction of Panc- 1 cell viability compared to the gemcitabine treatment alone (Fig. 4A). To investigate whether catechol and gemcitabine had synergistic cytotoxic effects in Panc-1 cells, combination index (CI) values were calculated. The $\mathrm{CI}$ values for drug combinations in Panc-1 cells ranged from 0.43 to 0.68. Combined treatment comprising $50 \mu \mathrm{M}$ of catechol and $0.25 \mu \mathrm{M}$ of gemcitabine exerted the highest synergistic inhibitory effects with a CI value of 0.43 (Fig. 4B). In addition, as revealed in Fig. 4C, condensed and fragmented chromatin and apoptotic bodies (arrows) were prominent in the combined-treatment groups. As the combined treatment ( $50 \mu \mathrm{M}$ of catechol and $0.25 \mu \mathrm{M}$ of gemcitabine) demonstrated the highest synergistic inhibitory effects, this combination was used in the following experiments to explore the mechanisms by which catechol synergizes with gemcitabine in Panc-1 cells. Cell cycle analysis revealed that sub-G1 populations of Panc-1 cells exposed to catechol and gemcitabine increased in a dose-dependent manner (Fig. 4D). The largest sub-G1 population $(10.05 \pm 4.40 \%)$ of Panc-1 cells was observed in the treatment combining $50 \mu \mathrm{M}$ of catechol and $0.25 \mu \mathrm{M}$ of gemcitabine (Fig. 4D). Furthermore, the combined treatment increased p-AMPK expression, cleavage of PARP, and the Bax/Bcl-2 ratio, as well as decreased YAP, CYR61, and p-STAT3 levels (Fig. 4E). Based on these observations, it was concluded that $50 \mu \mathrm{M}$ of catechol can enhance the in vitro cytotoxic efficacy of gemcitabine and promote gemcitabine-induced apoptosis in Panc-1 cells.

Catechol enhances the radiosensitivity of Panc-1 cells. The response of Panc-1 cells to radiation treatment in the presence or absence of catechol was examined by performing a colony formation assay. Results of the colony formation assay revealed that Panc-1 cell survival rates markedly decreased after exposure to catechol and radiation treatments (Fig. 5A). The linear-quadratic model was applied to generate radiosensitivity curves for each experimental group to represent the results of the colony formation assay (Fig. 5B). Catechol-treated cells were revealed to be more sensitive to radiation compared to control cells (Fig. 5B). Cell cycle analysis (Fig. 5C) revealed that combined catechol-exposure $(50 \mu \mathrm{M})$ and radiation treatment (4 Gy) increased the sub-G1 population of Panc-1 cells compared to the control (radiation only). Furthermore, western blotting revealed that the co-treatment group $(50 \mu \mathrm{M}$ catechol and 4 Gy radiation dose) had higher expression levels of p-ATM, p-Chk2, and cleaved PARP, known markers for radiation-induced DNA damage (20), compared to the untreated control (Fig. 5D). Increased AMPK levels have been reported to be associated with enhanced radiosensitivity (21). In the 

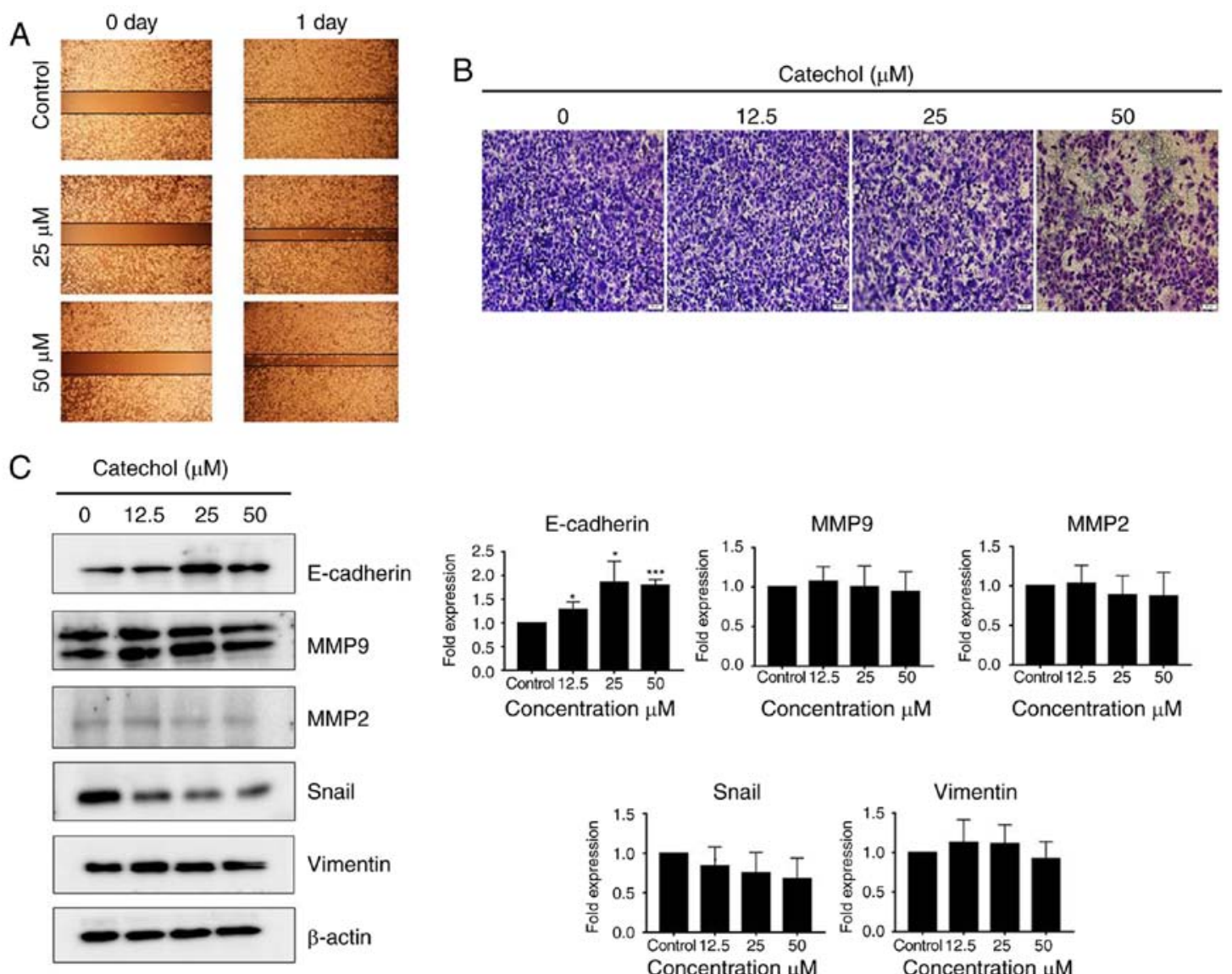

Figure 2. Catechol inhibits EMT in Panc-1 cells. (A) Effects of catechol on Panc-1 cell migration as determined by the wound-healing assay. (B) Invasion assay with Panc-1 cells after exposure to catechol for $24 \mathrm{~h}$. (C) Results of western blot analysis for EMT-related proteins after treatment with catechol for $24 \mathrm{~h}$. $\beta$-actin was used as the loading control. Band intensities were measured using ImageJ software (version 1.48). Values represent the means \pm SDs ( $n=3)$. "P<0.05 and ${ }^{* * * *} \mathrm{P}<0.001$ vs. the control. EMT, epithelial-mesenchymal transition; SDs, standard deviations.
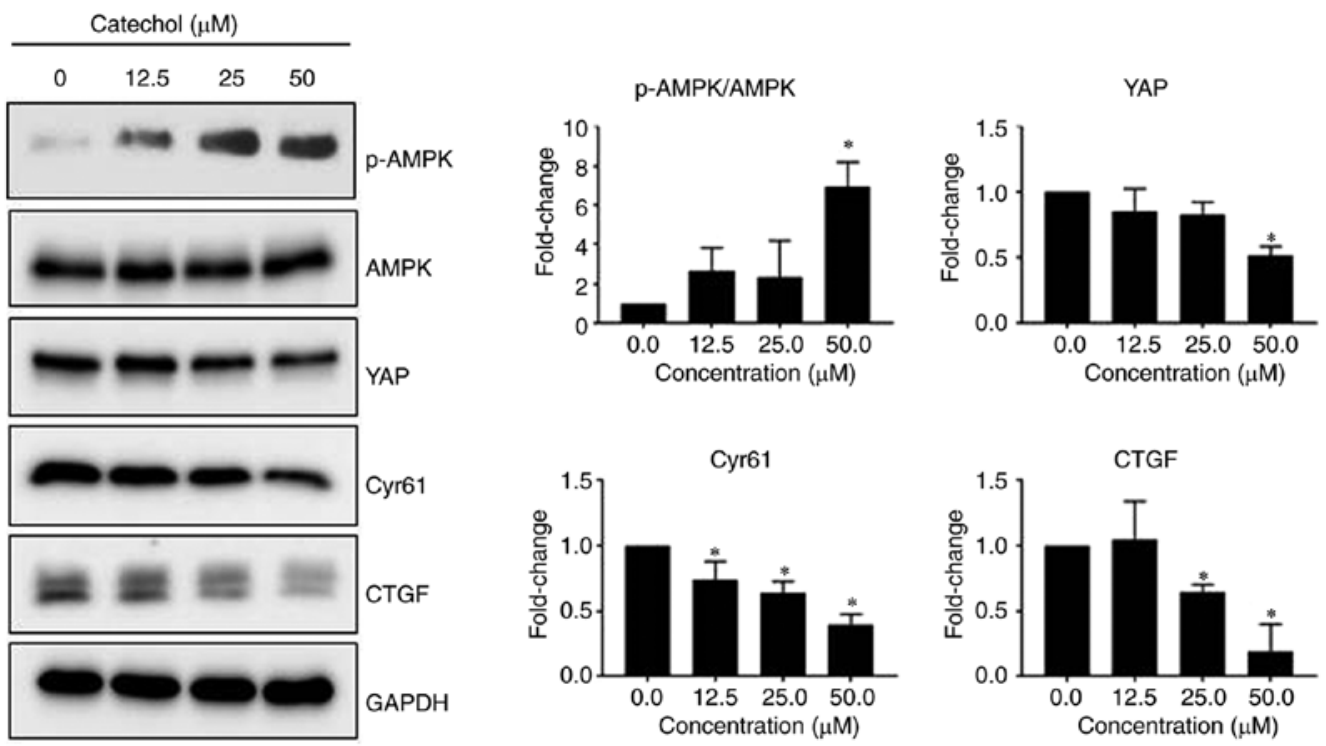

Figure 3. Catechol inhibits YAP expression through AMPK activation in Panc-1 cells. Results of western blot analysis of AMPK and YAP signaling pathway proteins after exposure to catechol for $48 \mathrm{~h}$. GAPDH was used as the loading control. Band intensities were measured using ImageJ software (version 1.48) Values represent the means $\pm \mathrm{SD}(\mathrm{n}=3)$. " $\mathrm{P}<0.05$ vs. the control. YAP, yes-associated protein; AMPK, AMP-activated protein kinase; p-, phosphorylated; CYR61, cysteine-rich angiogenic inducer 61; CTGF, connective tissue growth factor; SD, standard deviation.

present results, increased p-AMPK levels in the combined treatment group were observed, indicating that catechol could promote radiosensitivity by activating $\mathrm{p}$-AMPK. Moreover, the expression of YAP and CYR61 was reduced in the combined 
A

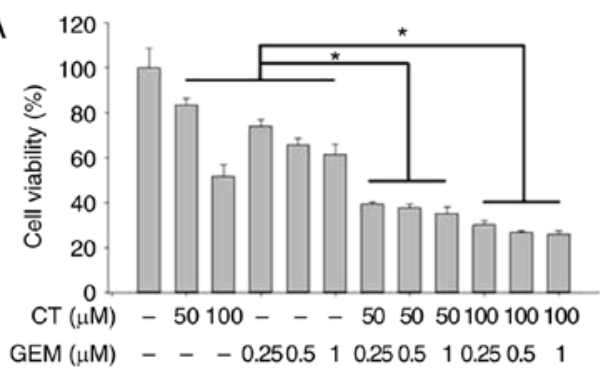

C

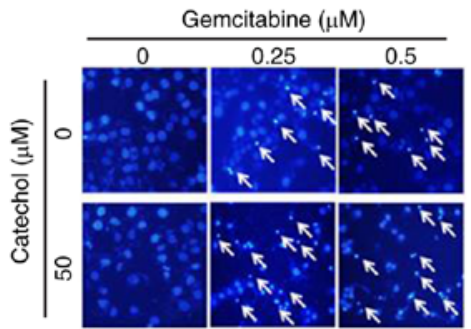

E

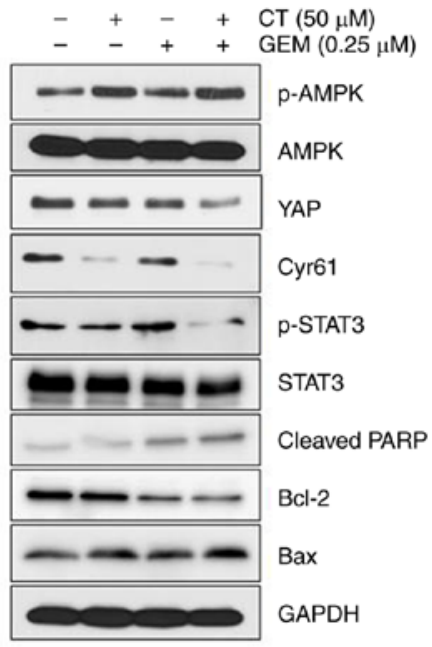

B

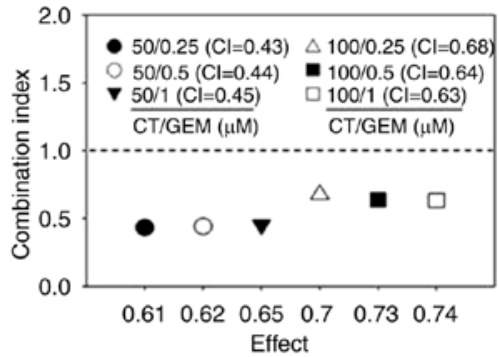

D

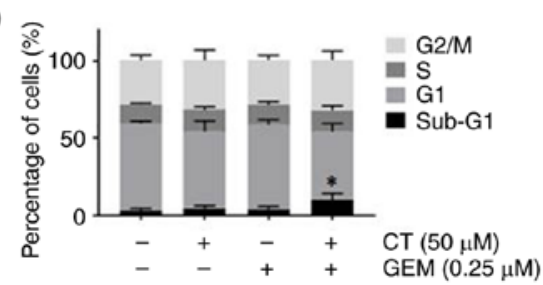

Figure 4. Catechol enhances the chemosensitivity of Panc-1 cells to gemcitabine. (A) Synergistic anti-proliferative effects of catechol and gemcitabine in Panc-1 cells as measured by the MTT assay. (B) CI values calculated for treatments exposing Panc-1 cells to combinations of various concentrations of catechol and gemcitabine. (C and D) Following exposure to $50 \mu \mathrm{M}$ of catechol, $0.25 \mu \mathrm{M}$ of gemcitabine, $0.5 \mu \mathrm{M}$ of gemcitabine, or catechol plus gemcitabine for $48 \mathrm{~h}$, Hoechst 33342 staining and cell cycle analysis were carried out. (E) Protein levels were examined using western blot analysis after exposing Panc-1 cells to catechol. GAPDH was used as the loading control. Band intensities were measured using ImageJ software (version 1.48). Values represent the means \pm SDs ( $=3$ ). ${ }^{*} \mathrm{P}<0.05$ vs. the control. CT, catechol; GEM, gemcitabine; CI, combination index; SDs, standard deviations; AMPK, AMP-activated protein kinase; p-, phosphorylated; YAP, yes-associated protein; CYR61, cysteine-rich angiogenic inducer 61; CTGF, connective tissue growth factor; PARP, poly(ADP-ribose) polymerase.

treatment group (Fig. 5D). These results indicated that catechol enhanced the effects of radiation treatments in Panc-1 cells.

\section{Discussion}

Pancreatic cancer is expected to become the third major cause of cancer-related deaths, exceeding deaths due to breast cancer, in most developed countries (22). Although chemotherapy, radiotherapy, and surgery can control pancreatic cancer metastasis to some extent, there is still no permanent cure. Clinically, resistance to gemcitabine after treatment initiation has been identified as a major therapeutic drawback in pancreatic cancer treatments $(6,7)$. In several pancreatic cancer clinical studies, the combination of gemcitabine with other chemotherapeutics has been revealed to improve the overall efficacy of gemcitabine vs. using the drug on its own $(23,24)$. Radiation therapy is often used in adjuvant or neoadjuvant settings in pancreatic cancer treatment. As in chemotherapy, adverse side effects and development of radiotherapy resistance have been identified as major clinical issues $(3,5)$.

Numerous herbal remedies have been used to treat various diseases and conditions for centuries. Some studies have revealed that numerous cancer patients use herbal medicine as complementary and alternative therapies (25). Various plant-derived herbal drugs or compounds have been reported to improve the efficacy of chemotherapeutics and decrease adverse side effects in cancer patients (25). Catechol (pyrocatechol) is a natural compound found in some fruits and vegetables. Lim et al (12) reported that catechol could target the ERKs/c-Myc signaling pathway in lung cancer in vitro and in vivo. Another study by Choi et al (11) reported that catechol targets breast cancer stem cells by dysregulating the Stat3/IL-6 survival pathway. In the present study, it was revealed that catechol was most effective at inhibiting the proliferation of Panc-1 cells among the four pancreatic cancer cell lines tested. Moreover, it was determined that 

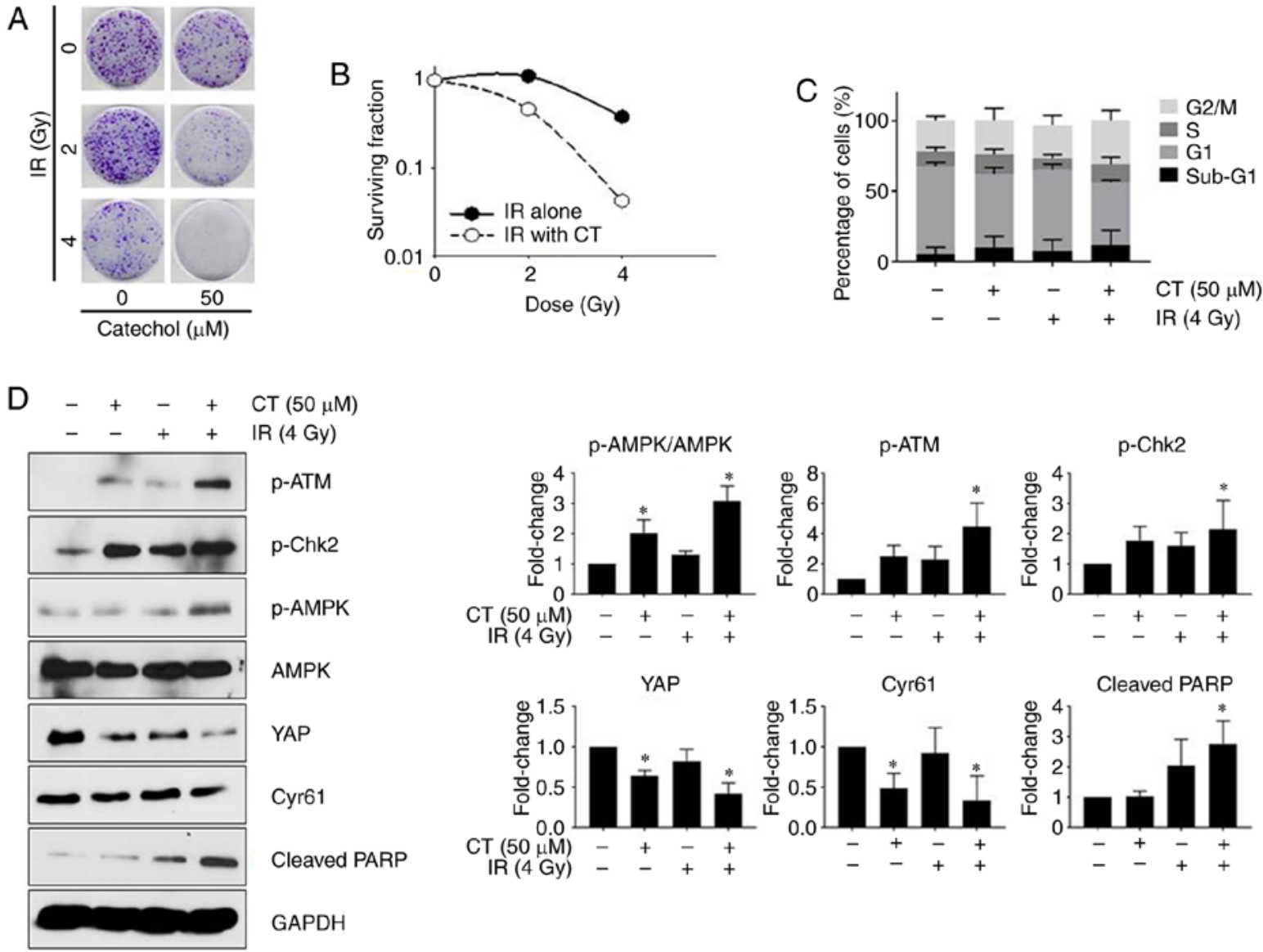

Figure 5. Catechol enhances the radiosensitivity of Panc-1 cells. (A) Clonogenic assay after treatment with catechol (CT; $50 \mu \mathrm{M})$ and radiation (IR; 2 or $4 \mathrm{~Gy}$ ). (B) The results obtained from the clonogenic assay. (C) Cell cycle analysis of Panc-1 cells following treatment with (50 $\mu \mathrm{M})$ or without CT and IR (4 Gy). (D) Protein levels in Panc-1 cells were examined using western blot analysis with GAPDH as the loading control. Band intensities were measured using ImageJ software (version 1.48). Values represent the means \pm SDs ( $n=3)$. ${ }^{*} \mathrm{P}<0.05$ vs. the control. CT, catechol; IR, radiation; SDs, standard deviations; AMPK, AMP-activated protein kinase; p-, phosphorylated; YAP, yes-associated protein; CYR61, cysteine-rich angiogenic inducer 61; CTGF, connective tissue growth factor; PARP, poly(ADP-ribose) polymerase.

catechol induced Panc-1 cell cycle arrest at the sub-G1 phase in a dose-dependent manner. In addition, western blot experiments confirmed that catechol could induce apoptosis in Panc-1 cells. EMT plays a key role in tumorigenesis and the development of chemo- and radio-therapy resistance (26). It was revealed that catechol reduced cell migration and invasion of Panc-1 cells and suppressed the expression of EMT-related markers such as Snail, vimentin, and MMP2, indicating the ability of catechol to inhibit Panc-1 cell migration and invasion by reducing the expression of EMT-associated proteins. The Hippo signaling pathway plays a regulatory role in tumorigenesis (27). The transcription co-activator YAP in Hippo signaling is often overexpressed in a range of human cancers, making it an attractive pharmacological target 27). Activated AMPK-mediated YAP inhibition has been reported in previous investigations $(19,28)$. Moreover, studies have revealed that phytochemicals such as curcumin and resveratrol can target YAP in breast and bladder cancer cells $(29,30)$. In the present investigation, catechol was revealed to increase the expression of $\mathrm{p}$-AMPK with a concomitant decrease in the levels of CTGF and CYR61, two main downstream targets of YAP in Hippo signaling, suggesting that catechol can function as a new drug lead that targets Hippo signaling in Panc-1 cells.
As resistance to gemcitabine is frequently observed in pancreatic cancer patients, new approaches to improve the clinical efficacy of gemcitabine in pancreatic cancer treatments should be explored. Treatment using chemotherapeutics combined with natural drugs has been reported to enhance the overall efficacy of chemotherapy treatments as well as minimize adverse side effects (31). In the present study, it was demonstrated that catechol enhanced both the chemosensitivity of Panc-1 cells to gemcitabine and gemcitabine-induced apoptosis, which were confirmed by cell viability assays, flow cytometry, and western blot experiments. A decreased sensitivity of pancreatic tumors to radiation therapy significantly influences the life expectancy of pancreatic cancer patients $(6,7)$. Several natural radio-sensitizers have been revealed to enhance the overall efficacy of radiation treatment in a range of human cancers (32). Notably, it was observed that catechol improved radiosensitivity in Panc-1 cells. Moreover, the results of western blot experiments confirmed that radiation therapy along with catechol increased the expression of radiation-induced DNA-damage markers, including p-ATM, p-Chk2, p-AMPK, and cleaved PARP, in Panc-1 cells, indicating the potential use of catechol/radiation combined therapy for pancreatic cancer patients. Considering the importance of ATM serine/threonine kinase (ATM) in DNA 
double strand breaks (DSBs) in response to IR, the effects of catechol on the expression of ATM-associated pathway proteins were assessed. Activation of ATM occurs by auto-phosphorylation at Ser1981 upon DNA damage induced by IR. Following DNA damage, ATM can phosphorylate Chk2 at Thr68 (33). Hence, considering ATM and Chk2 as markers for DNA damage in response to IR, the activated forms (phosphorylated forms) of ATM and Chk2 were only used (33).

In conclusion, catechol induced apoptosis and cell cycle arrest and suppressed the expression of EMT-related markers in Panc-1 cells. Above all, catechol enhanced the chemosensitivity of Panc-1 cells to gemcitabine and induced radiation sensitization in Panc-1 cells, indicating that catechol can play a prominent role in overcoming chemo- and radio-resistance, a major clinical challenge in pancreatic cancer. Additionally, catechol was revealed to modulate the AMPK/Hippo signaling pathway in pancreatic cancer cells. Results of the present study provide a strong rationale for exploring the clinical efficacy of catechol in the treatment of pancreatic cancer. We are currently investigating the in vivo efficacy of catechol in nude mice xenografts and the results will be published in future. Moreover, we are currently planning to initiate new experiments to investigate the effects of catechol to assess the loss- and gain-of-function of STAT-3.

\section{Acknowledgements}

Not applicable.

\section{Funding}

The present research was supported by the 2020 Scientific Promotion Program funded by Jeju National University, the National Research Foundation of Korea (NRF) grant no. 2020R1A2C1004349, and by the Basic Science Research Program through the NRF funded by the Ministry of Education (grant no. 2016R1A6A1A03012862).

\section{Availability of data and materials}

The datasets used and/or analyzed during the current study are available from the corresponding author upon reasonable request.

\section{Authors' contributions}

JYM and SKC designed the study. JYM, MKE, JYR and HYK performed experiments. JYM and MKE analyzed the data and wrote the manuscript. SKC supervised the study and revised the manuscript. All authors read and approved the final manuscript.

\section{Ethics approval and consent to participate}

Not applicable.

\section{Patient consent for publication}

Not applicable.

\section{Competing interests}

The authors declare that they have no competing interests.

\section{References}

1. Bray F, Ferlay J, Soerjomataram I, Siegel RL, Torre LA and Jemal A: Global cancer statistics 2018: GLOBOCAN estimates of incidence and mortality worldwide for 36 cancers in 185 countries. CA Cancer J Clin 68: 394-424, 2018.

2. Siegel RL, Miller KD and Jemal A: Cancer statistics, 2019. CA Cancer J Clin 69: 7-34, 2019.

3. Rawla P, Sunkara T and Gaduputi V: Epidemiology of pancreatic cancer: Global trends, etiology and risk factors. World J Oncol 10: 10-27, 2019.

4. Collisson EA, Bailey P, Chang DK and Biankin AV: Molecular subtypes of pancreatic cancer. Nat Rev Gastroenterol Hepatol 16: 207-220, 2019.

5. Li D, Xie K, Wolff R and Abbruzzese JL: Pancreatic cancer. Lancet 363: 1049-1057, 2004.

6. Amrutkar M and Gladhaug IP: Pancreatic cancer chemoresistance to gemcitabine. Cancers (Basel) 9: 157, 2017.

7. Nakano Y, Tanno S, Koizumi K, Nishikawa T, Nakamura K, Minoguchi M, Izawa T, Mizukami Y, Okumura T and Kohgo Y: Gemcitabine chemoresistance and molecular markers associated with gemcitabine transport and metabolism in human pancreatic cancer cells. Br J Cancer 96: 457-463, 2007.

8. Strobel O, Neoptolemos J, Jaeger D and Buechler MW: Optimizing the outcomes of pancreatic cancer surgery. Nat Rev Clin Oncol 16: 11-26, 2019.

9. Newman DJ and Cragg GM: Natural products as sources of new drugs from 1981 to 2014. J Nat Prod 79: 629-661, 2016.

10. Ediriweera MK, Tennekoon KH and Samarakoon SR: In vitro assays and techniques utilized in anticancer drug discovery. J Appl Toxicol 39: 38-71, 2019.

11. Choi HS, Kim JH, Kim SL, Deng HY, Lee D, Kim CS, Yun BS and Lee DS: Catechol derived from aronia juice through lactic acid bacteria fermentation inhibits breast cancer stem cell formation via modulation Stat3/IL-6 signaling pathway. Mol Carcinog 57: 1467-1479, 2018.

12. Lim DY, Shin SH, Lee MH, Malakhova M, Kurinov I, Wu Q, Xu J, Jiang Y, Dong Z, Liu K, et al: A natural small molecule, catechol, induces c-Myc degradation by directly targeting ERK2 in lung cancer. Oncotarget 7: 35001-35014, 2016.

13. Tran TA, Ho MT, Song YW, Cho M and Cho SK: Camphor induces proliferative and anti-senescence activities in human primary dermal fibroblasts and inhibits UV-induced wrinkle formation in mouse skin. Phytother Res 12: 1917-1925, 2015.

14. Wang T, Luo R, Li W, Yan H, Xie S, Xiao W, Wang Y, Chen B, Bai P and Xing J: Dihydroartemisinin suppresses bladder cancer cell invasion and migration by regulating KDM3A and $\mathrm{p} 21$. J Cancer 11: 1115, 2020.

15. Tadros S, Shukla SK, King RJ, Gunda V, Vernucci E, Abrego J, Chaika NV, Yu F, Lazenby AJ, Berim L, et al: De novo lipid synthesis facilitates gemcitabine resistance through endoplasmic reticulum stress in pancreatic cancer. Cancer Res 77: 5503-5517, 2017.

16. Al Zaid Siddiquee K and Turkson J: STAT3 as a target for inducing apoptosis in solid and hematological tumors. Cell Res 18: 254-267, 2008.

17. Zhao B, Lei QY and Guan KL: The Hippo-YAP pathway: New connections between regulation of organ size and cancer. Curr Opin Cell Biol 20: 638-646, 2008.

18. Mo JS, Yu FX, Gong R, Brown JH and Guan KL: Regulation of the Hippo-YAP pathway by protease-activated receptors (PARs). Genes Dev 26: 2138-2143, 2012.

19. Mo JS, Meng Z, Kim YC, Park HW, Hansen CG, Kim S, Lim DS and Guan KL: Cellular energy stress induces AMPK-mediated regulation of YAP and the Hippo pathway. Nat Cell Biol 17: 500-510, 2015.

20. Hsu PC, Gopinath RK, Hsueh YA and Shieh SY: CHK2-mediated regulation of PARP1 in oxidative DNA damage response. Oncogene 38: 1166-1182, 2019.

21. Adeberg S, Bernhardt D, Harrabi SB, Nicolay $\mathrm{NH}$, Hörner-Rieber J, König L, Repka M, Mohr A, Abdollahi A, Weber KJ, et al: Metformin enhanced in vitro radiosensitivity associates with $\mathrm{G} 2 / \mathrm{M}$ cell cycle arrest and elevated adenosine-5'-monophosphate-activated protein kinase levels in glioblastoma. Radiol Oncol 51: 431-437, 2017. 
22. Ferlay J, Partensky $\mathrm{C}$ and Bray F: More deaths from pancreatic cancer than breast cancer in the EU by 2017. Acta Oncol 55: 1158-1160, 2016.

23. Infante JR, Somer BG, Park JO, Li CP, Scheulen ME, Kasubhai SM, Oh DY, Liu Y, Redhu S, Steplewski K and Le N: A randomised, double-blind, placebo-controlled trial of trametinib, an oral MEK inhibitor, in combination with gemcitabine for patients with untreated metastatic adenocarcinoma of the pancreas. Eur J Cancer 50: 2072-2081, 2014.

24. Le Chevalier T, Scagliotti G, Natale R, Danson S, Rosell R, Stahel R, Thomas P, Rudd RM, Vansteenkiste J, Thatcher N, et al Efficacy of gemcitabine plus platinum chemotherapy compared with other platinum containing regimens in advanced non-small-cell lung cancer: A meta-analysis of survival outcomes. Lung Cancer 47: 69-80, 2005.

25. Cassileth BR and Deng G: Complementary and alternative therapies for cancer. Oncologist 9: 80-89, 2004.

26. Davis FM, Stewart TA, Thompson EW and Monteith GR: Targeting EMT in cancer: Opportunities for pharmacological intervention. Trends Pharmacol Sci 35: 479-488. 2014.

27. Pan D: The hippo signaling pathway in development and cancer. Dev Cell 19: 491-505, 2010

28. DeRan M, Yang J, Shen CH, Peters EC, Fitamant J, Chan P, Hsieh M, Zhu S, Asara JM, Zheng B, et al: Energy stress regulates hippo-YAP signaling involving AMPK-mediated regulation of angiomotin-like 1 protein. Cell Rep 9: 495-503, 2014.
29. Gao Y, Shi Q, Xu S, Du C, Liang L, Wu K, Wang K, Wang X, Chang LS, He D and Guo P: Curcumin promotes KLF5 proteasome degradation through downregulating YAP/TAZ in bladder cancer cells. Int J Mol Sci 15: 15173-15187, 2014

30. Kim YN, Choe SR, Cho KH, Kang J, Park CG and Lee HY Resveratrol suppresses breast cancer cell invasion by inactivating a RhoA/YAP signaling axis. Exp Mol Med 49: e296-e296, 2017.

31. Nobili S, Lippi D, Witort E, Donnini M, Bausi L, Mini E and Capaccioli S: Natural compounds for cancer treatment and prevention. Pharmacol Res 59: 365-378, 2009.

32. Malik A, Sultana M, Qazi A, Qazi MH, Parveen G, Waquar S, Ashraf AB and Rasool M: Role of natural radiosensitizers and cancer cell radioresistance: An update. Anal Cell Pathol (Amst) 2016: 6146595, 2016.

33. Chen L, Gilkes DM, Pan Y, Lane WS and Chen J: ATM and Chk2-dependent phosphorylation of MDMX contribute to p53 activation after DNA damage. EMBO J 24: 3411-3422, 2005.

This work is licensed under a Creative Commons Attribution-NonCommercial-NoDerivatives 4.0 International (CC BY-NC-ND 4.0) License. 